\title{
Ideals on Intuitionistic Fuzzy Supra Topological Spaces
}

\section{Fadhil Abbas}

Salzburger Straße 195, Linz, Austria

\section{]jfis}

Received: Nov. 21, 2020

Revised : Jan. 29, 2021

Accepted: Mar. 3, 2021

Correspondence to: Fadhil Abbas

(fadhilhaman@gmail.com)

(The Korean Institute of Intelligent Systems

CCThis is an Open Access article distributed under the terms of the Creative Commons Attribution Non-Commercial License (http://creativecommons.org/licenses/by-nc / 3.0/) which permits unrestricted noncommercial use, distribution, and reproduction in any medium, provided the original work is properly cited.

\begin{abstract}
The purpose of this paper, is introduce the notion of ideals on intuitionistic fuzzy supra topological spaces. Also present the notion of S-compatible with the intuitionistic fuzzy ideal I and investigation some properties of intuitionistic fuzzy supra topological spaces $\mathrm{S}$ with the intuitionistic fuzzy ideal I. Moreover, introduce an intuitionistic fuzzy set operator $\Psi_{S}$ and study its properties.
\end{abstract}

Keywords: Intuitionistic fuzzy-I-supra topology, Intuitionistic fuzzy s-local function, Intuitionistic fuzzy set operator $\Psi_{S}$

\section{Introduction}

Zadeh [1] introduced the notion of fuzzy sets in 1965. Now, they are one of the most serious and possible paths for the advancement of the set theory of introduced by Georg Cantor. Despite the doubts and critical remarks expressed by some of the most influential mathematical logic experts in the second half of the 1960s against fuzzy sets, fuzzy sets were firmly developed as a fruitful field of study as well as a method for evaluating various objects and procedures.

In 1986, Atanassov [2] introducedintroduced intuitionistic fuzzy sets. In many applications, the intuitionist fuzzy sets are important and useful fuzzy sets. Atanassov [3, 4] in 1994 and 1999 proved that the intuitionistic fuzzy sets contain the degree of affiliation and the degree of non-affiliation, and therefore, the intuitionistic fuzzy sets have become more relevant and applicable. In 2001 and 2004, Szmidt and Kacprzyk [5, 6] showed that intuitionist fuzzy sets are so useful in situations where it seems extremely difficult to define a problem through a membership function.

The idea of intuitionistic fuzzy topology was described by Atanassov [7] in 1988, and the basic idea of intuitionistic fuzzy points was studied by Coker and Demirci [8] in 1995. Kuratowski [9] first proposed the concept of an ideal topological space in 1966, and Vaidyanathaswamy [10] proposed in 1944. In an ideal topological space, they also introduced a local function. In 1990, Jankovic and Hamlett [11] introduced a new topology by introduce the operator in any ideal topological space from the original ideal topological spaces.

Mashhour et al. [12] in 1983 introduced supra topological spaces. The concept of intuitionist fuzzy supra topological space was introduced by Turanl [13] in 2001. In addition to some features of an ideal supra topological notion obtained by Kandil et al. [14] in 2015.

The purpose of this paper is to introduce the notion of ideals on intuitionistic fuzzy supra topological spaces. Also, present the notion of $\mathrm{S}$ is compatible with the intuitionistic fuzzy 
ideal I and investigation some properties of intuitionistic fuzzy supra topological spaces $\mathrm{S}$ with intuitionistic fuzzy ideal $\mathrm{I}$. Moreover, introduce an intuitionistic fuzzy set operator $\Psi_{S}$ and study its properties.

\section{Preliminaries}

Definition 2.1( [15]). Let $X \neq \emptyset$, an intuitionistic fuzzy set $\mathrm{A}$ is subject with the form $A=\left\{\left\langle x, \mu_{A}(x), \nu_{A}(x)\right\rangle\right.$ : $x \in X\}$, where $\mu_{A}: X \longrightarrow[0,1]$ and $\nu_{A}: X \longrightarrow[0,1]$ define the degree of membership $\mu_{A}(x)$ and the degree of nonmembership $\nu_{A}(x)$ for every $x \in X$ to the set A, respectively, and $0 \leqslant \mu_{A}(x)+\nu_{A}(x) \leq$ for every $x \in X$.

Definition $2.2([15]) .1_{\sim}=\{\langle x, 1,0\rangle: x \in X\}$ and $0_{\sim}=\{\langle x, 0,1\rangle: x \in X\}$.

Definition 2.3 ( [16]). Let A, B be an intuitionistic fuzzy sets, then we define

1. $A \subseteq B$ if and only if $\mu_{A}(x) \leqslant \mu_{B}(x)$ and $\nu_{A}(x) \geq$ $\nu_{B}(x)$ for every $x \in X$.

2. $A=B$ if and only if $A \subseteq B$ and $B \subseteq A$.

3. $\left.A^{c}=\left\{<x, \nu_{A}(x), \mu_{A}(x): x \in X\right\rangle\right\}$.

4. $A \cap B=\left\{<x, \mu_{A}(x) \wedge \mu_{B}(x), \mu_{A}(x) \vee \mu_{B}(x)>\right\}$.

5. $A \cup B=\left\{<x, \mu_{A}(x) \vee \mu_{B}(x), \mu_{A}(x) \wedge \mu_{B}(x)>\right\}$.

Definition 2.4 ( $|8|)$. Let $X \neq \emptyset$ and let $x \in X$. If $\alpha \in(0,1]$ and $\beta \in[0,1)$ are two fixed real numbers such that $\alpha+\beta \leqslant 1$, then, in the intuitionistic fuzzy set

$$
x_{(\alpha, \beta)}=\left\{<x, x_{(\alpha)}, 1-x_{(\beta)}>: x \in X\right\}
$$

is called an intuitionistic fuzzy point in $\mathrm{X}$, where $\alpha$ denotes the degree of membership of $x_{(\alpha, \beta)}, \beta$ is the degree of nonmembership of $x_{(\alpha, \beta)}$, and $x \in X$ is the support of $x_{(\alpha, \beta)}$.

Definition 2.5 ( [2]). A subclass $\mathrm{S}$ is called an intuitionistic fuzzy supra topology on $\mathrm{X}$ if $0_{\sim}, 1_{\sim} \in S$ and $\mathrm{S}$ is closed under arbitrary unions $(X, S)$, which is called an intuitionistic fuzzy supra topoloical topological space, the members of $\mathrm{S}$ are called intuitionistic fuzzy supra open sets. An intuitionistic fuzzy set $\mathrm{A}$ is an intuitionistic fuzzy supra closed if and only if its complement $A^{c}$ is fuzzy supra open.

Definition 2.6 ( [2]). Let (X, S) be an intuitionistic fuzzy supra topological space and let A be an intuitionistic fuzzy set in X. ThenSubsequently, the intuitionistic fuzzy supra interior and the intuitionistic fuzzy supra closure of $\mathrm{A}$ in $(\mathrm{X}, \mathrm{S})$ is defined as

$$
\operatorname{Int}^{S}(A)=\bigcup\{U: U \subseteq A, U \in S\},
$$

and

$$
C l^{S}(A)=\bigcap\left\{F: A \subseteq F, F^{c} \in S\right\},
$$

respectively.

Corollary 2.1. From Definition 2.6, $\operatorname{Int}^{S}(A)$ is a fuzzy supra open set, and $C l^{S}(A)$ is a fuzzy supra closed set.

Definition 2.7 ( $[8])$. Let A and B be two intuitionistic fuzzy sets in X. A is called quasi-coincident with B (written AqB) if and only if, there exists $x \in X$ such that $\mu_{A}(x)>\nu_{B}(x)$ or $\nu_{A}(x)<\mu_{B}(x)$.

Definition $2.8([8])$. Let $x_{(\alpha, \beta)}$ an intuitionistic fuzzy point and let $\mathrm{A}$ an intuitionistic fuzzy set in $\mathrm{X}$. We say that $x_{(\alpha, \beta)}$ quasi-coincident with $\mathrm{A}$, denoted by $x_{(\alpha, \beta)} \mathrm{qA}$ if and only if $\alpha>\nu_{A}(x)$ or $\beta<\mu_{A}(x)$.

Definition 2.9 ([8]). Let $x_{(\alpha, \beta)}$ an intuitionistic fuzzy point and let $\mathrm{A}$ an intuitionistic fuzzy set in X. Let $\alpha$ and $\beta$ are real numbers between 0 and 1 . The intuitionistic fuzzy point $x_{(\alpha, \beta)}$ is called properly contained in A if and only if, $\alpha<\mu_{A}(x)$ and $\beta>\nu_{A}(x)$.

Definition $2.10(|\overline{8}|)$. Let $x_{(\alpha . \beta)}$ an intuitionistic fuzzy point . Then, $x_{(\alpha, \beta)} \in A$ if $\alpha \leq \mu_{A}(x)$ and $\beta \geq \nu_{A}(x)$.

Definition 2.11 ( [2]). Let (X, S) be an intuitionistic fuzzy supra topological space and let $A \subseteq X$. Then, $\mathrm{A}$ is the sneighborhood of an intuitionistic fuzzy point $x_{(\alpha, \beta)}$ if there is $U \in S$ with $x_{(\alpha, \beta)} \in U \subseteq A\left(x_{(\alpha, \beta)} q U \subseteq A\right)$. The collection $N\left(x_{(\alpha, \beta)}\right)$ of all s-neighborhood of $x_{(\alpha, \beta)}$ is called the s-neighborhood system of $x_{(\alpha, \beta)}$.

Definition 2.12 ( [2]). Let $\left(X, S_{1}\right)$ and $\left(X, S_{2}\right)$ be two intuitionistic fuzzy supra topologies and let $S_{1} \subseteq S_{2}$. Then, we say that $S_{2}$ is stronger than $S_{1}$ or $S_{1}$ is weaker than $S_{2}$.

Definition 2.13 ( [2]). Let $(X, S)$ be an intuitionistic fuzzy supra topological space and let $\beta \subseteq S$. Then, $\beta$ is called a base for the intuitionistic fuzzy supra topology $S$ if every intuitionistic fuzzy supra open set $U \in S$ is a union of members of $\beta$. Equivalently, $\beta$ is an intuitionistic fuzzy supra-base for $\mathrm{S}$ if for any intuitionistic fuzzy point $x_{(\alpha, \beta)} \in U$ there exists $B \in \beta$ with $x_{(\alpha, \beta)} \in B \subseteq U$.

Definition 2.14 ([2]). A mapping $c: P(X) \rightarrow P(X)$ is called an intuitionistic fuzzy supra closure operator if it satisfies 
the following axioms:

1. $c\left(0_{\sim}\right)=0_{\sim}$,

2. $A \subseteq c(A)$ for every $A \subseteq X$,

3. $c(A) \cup c(B) \subseteq c(A \cup B)$ for every $A, B \subseteq X$,

4. $c(c(A))=c(A)$ for every $A \subseteq X$.

Theorem 2.1 ([2]). Let $X \neq \emptyset$ and let the mapping $c: P(X)$ $\rightarrow P(X)$ be an intuitionistic fuzzy supra closure operator. Then, the collection $S=\left\{A \in P(X): c\left(A^{c}\right)=A^{c}\right\}$ is an intuitionistic fuzzy supra topology on $\mathrm{X}$ induced by the intuitionistic fuzzy supra closure operator $\mathrm{c}$.

Definition 2.15 ( [15]). Let I be a non-empty collection of intuitionistic fuzzy sets of $\mathrm{X}$ is called intuitionistic fuzzy ideal on $\mathrm{X}$ if and only if

1. $A \in I$ and $B \subseteq A$, then $B \in I$,

2. $A \in I$ and $B \in I$, then $A \cup B \in I$.

\section{Intuitionistic Fuzzy S-Local Function}

Definition 3.1. Let $(X, S)$ be an intuitionistic fuzzy supra topological space. Then, an intuitionistic fuzzy ideal I on $\mathrm{X}$ is called an intuitionistic fuzzy ideal supra topological space and is denoted as $(\mathrm{X}, \mathrm{S}, \mathrm{I})$.

Definition 3.2. Let (X, S, I) be an intuitionistic fuzzy ideal supra topological space and let A be an intuitionistic fuzzy set in $\mathrm{X}$. Then, the intuitionistic fuzzy S-local function $A^{* S}(I, S)$ of A is the union of all intuitionistic fuzzy point $x_{(\alpha, \beta)}$ such that if $U \in N\left(x_{(\alpha, \beta)}\right)$ and $A^{* S}(I, S)=\cup\left\{x_{(\alpha, \beta)} \in X: A \cap U \notin I\right.$, for every $\left.U \in N\left(x_{(\alpha, \beta)}\right)\right\}$. We will occasionally write $A^{* S}$ for $A^{* S}(I, S)$.

Example 3.1. The simplest intuitionistic fuzzy ideal on $\mathrm{X}$ isare $\{0 \sim\}$ and $P(X)$. Obviously, $I=\left\{0_{\sim}\right\} \Leftrightarrow A^{* S}=$ $C l^{S}(A)$, for any $A \subseteq X$ and $I=P(X) \Leftrightarrow A^{* S}=0_{\sim}$.

Theorem 3.1. Let (X, S, I) be an intuitionistic fuzzy supra topological space and let $A, B \subseteq X$. Then,

(1) $0_{\sim}^{* S}=0_{\sim}$,

(2) If $A \subseteq B$, then $A^{* S} \subseteq B^{* S}$,

(3) If $I_{1} \subseteq I_{2}$, then $A^{* S}\left(I_{2}\right) \subseteq A^{* S}\left(I_{1}\right)$,

(4) $A^{* S}=C l^{S}\left(A^{* S}\right) \subseteq C l^{S}(A)$,

(5) $\left(A^{* S}\right)^{* S} \subseteq A^{* S}$,
(6) $A^{* S}$ is an intuitionistic fuzzy supra closed set,

(7) $A^{* S} \cup B^{* S} \subseteq(A \cup B)^{* S}$,

(8) $(A \cap B)^{* S} \subseteq A^{* S} \cap B^{* S}$,

(9) If $E \in I$, then $(A \cup E)^{* S}=A^{* S}=(A-E)^{* S}$,

(10) If $U \in S$, then $U \cap A^{* S}=U \cap(U \cap A)^{* S} \subseteq(U \cap A)^{* S}$,

(11) If $E \in I$, then $E^{* S}=0_{\sim}$,

(12) $E \in I$, then $\left(1_{\sim}-E\right)^{* S}=1_{\sim}^{* S}$.

Proof. (1) Clear from the definition of intuitionistic fuzzy Slocal function .

(2) Since $A \subseteq B$, let $x_{(\alpha, \beta)} \in A^{* S}$, then $A \cap U \notin I$ for every $U \in N\left(x_{(\alpha, \beta)}\right)$. By hypothesis, we obtainget $B \cap U \notin I$, then $x_{(\alpha, \beta)} \in B$. Therefore, $A^{* S} \subseteq B^{* S}$.

(3) Cleary, $I_{1} \subseteq I_{2}$ implies $A^{* S}\left(I_{2}\right) \subseteq A^{* S}\left(I_{1}\right)$, as there may be other intuitionistic fuzzy sets that which belong to $I_{2}$ so that for an ituitionistic fuzzy point $x_{(\alpha, \beta)} \in A^{* S}\left(I_{1}\right)$ but $x_{(\alpha, \beta)} \notin A^{* S}\left(I_{2}\right)$.

(4) Since $\left\{0_{\sim}\right\} \subseteq I$ for any intuitionistic fuzzy ideal on $X$, therefore by (3) and Example 3.1. $A^{* S}(I) \subseteq A^{* S}\left(\left\{0_{\sim}\right\}\right)=$ $C l^{S}(A)$, for any intuitionistic fuzzy set A in X. Suppose, $x_{1(\alpha, \beta)}$ $\in C l^{S}\left(A^{* S}\right)$ such that for every $U \in N\left(x_{1(\alpha, \beta)}\right), A^{* S} \cap U \neq$ $0 \sim$ there exists $x_{2(\alpha, \beta)} \in A^{* S} \cap U$ such that for every $V \in$ $N\left(x_{2(\alpha, \beta)}\right)$, then $A \cap V \notin I$. Since $U \cap V \in N\left(x_{2(\alpha, \beta)}\right)$, then $A \cap(U \cap V) \notin I$, which leads to $A \cap U \notin I$ for every $U \in N\left(x_{1(\alpha, \beta)}\right)$, therefore $x_{1(\alpha, \beta)} \in A^{* S}$; and so $C l^{S}\left(A^{* S}\right) \subseteq A^{* S}$ while the other inclusion follows directly. Hence, $A^{* S}=C l^{S}\left(A^{* S}\right) \subseteq C l^{S}(A)$.

(5) From (4), $\left(A^{* S}\right)^{* S} \subseteq C l^{S}\left(A^{* S}\right)=A^{* S}$.

(6) Clear from (4).

(7) We know that $A \subseteq A \cup B$ and $B \subseteq A \cup B$. Then, from (2), $A^{* S} \subseteq(A \cup B)^{* S}$ and $B^{* S} \subseteq(A \cup B)^{* S}$. Hence, $A^{* S} \cup B^{* S} \subseteq(A \cup B)^{* S}$.

(8) We know that $(A \cap B) \subseteq A$ and $(A \cap B) \subseteq B$. Then, from (2), $(A \cap B)^{* S} \subseteq A^{* S}$ and $(A \cap B)^{* S} \subseteq A^{* S}$. Hence, $(A \cap B)^{* S} \subseteq A^{* S} \cap B^{* S}$.

(9) Since $A \subseteq(A \cup E)$, then from (2) $A^{* S} \subseteq(A \cup E)^{* S}$ Let $x_{(\alpha, \beta)} \in(A \cup E)^{* S}$. Then, for every $U \in N\left(x_{(\alpha, \beta)}\right)$ such that $U \cap(A \cup E) \notin I$. This implies that $U \cap A \notin I$ (if possible suppose that $U \cap A \in I$. Again, $U \cap E \subseteq E$ implies $U \cap E \in I$ and hence $U \cap(A \cup E) \in I$, contradiction). Hence, $x_{(\alpha, \beta)} \in A^{* S}$ and $(A \cup E)^{* S} \subseteq A^{* S}$ then $(A \cup E)^{* S}=A^{* S}$.

Since $(A-E) \subseteq A$, then from (2), $(A-E)^{* S} \subseteq A^{* S}$. For the reverse inclusion, let $x_{(\alpha, \beta)} \in A^{* S}$. We claim that 
$x_{(\alpha, \beta)} \in(A-E)^{* S}$, if not, then there is $U \in N\left(x_{(\alpha, \beta)}\right)$ such that $U \cap(A-E) \in I$. Given that $E \in I$, then $E \cup$ $(U \cap(A-E)) \in I$. This implies that $E \cup(U \cap A) \in I$. So, $U \cap A \in I$, a contradiction to the fact that $x_{(\alpha . \beta)} \in A^{* S}$. Hence, $A^{* S} \subseteq(A-E)^{* S}$. Then, $A^{* S}=(A-E)^{* S}$; therefore, $(A \cup E)^{* S}=A^{* S}=(A-E)^{* S}$.

(10) Since $V \cap A \subseteq A$, then from (2), $(V \cap A)^{* S} \subseteq A^{* S}$. So $V \cap(V \cap A)^{* S} \subseteq V \cap A^{* S}$.

(11) Clear from the definition of intuitionistic fuzzy S-local function.

(12) Clear from proof (9).

Theorem 3.2. Let (X, S, I) be an intuitionistic fuzzy ideal supra topological space and let $A \subseteq X$. If $M \in S, M \cap A \in I$, then $M \cap A^{* S}=0_{\sim}$.

Proof. Let $x_{(\alpha, \beta)} \in M \cap A^{* S}$. Then, $x_{(\alpha, \beta)} \in M$ and $x_{(\alpha, \beta)} \in$ $A^{* S}$ implies $U \cap A \notin I$ for every $U \in N\left(x_{(\alpha, \beta)}\right)$. Since $x_{(\alpha, \beta)} \in M \in S$, then $M \cap A^{* S} \notin I$.

Theorem 3.3. Let $(X, S, I)$ be an intuitionistic fuzzy ideal supra topological space and let $A \subseteq X$. Then, $\left(A \cup A^{* S}\right)^{* S} \subseteq$ $A^{* S}$.

Proof. Let $x_{(\alpha, \beta)} \notin A^{* S}$. Then, there exists $U \in N\left(x_{(\alpha, \beta)}\right)$ such that $U \cap A \in I \Rightarrow U \cap A^{* S}=0_{\sim}$ (By Theorem 3.2.). Hence, $U \cap\left(A \cup A^{* S}\right)=(U \cap A) \cup\left(U \cap A^{* S}\right)=U \cap A \in I$. Therefore, $x_{(\alpha, \beta)} \notin\left(A \cup A^{* S}\right)^{* S}$. Hence, $\left(A \cup A^{* S}\right)^{* S} \subseteq$ $A^{* S}$.

Theorem 3.4. Let $(X, S, I)$ be an intuitionistic fuzzy ideal supra topological space. Then, the operator $C l^{* S}: P(X) \longrightarrow$ $P(X)$ defined by $C l^{* S}(A)=A \cup A^{* S}$ for any $A \subseteq X$, is an intuitionistic fuzzy supra closure operator and hence it generates an intuitionistic fuzzy supra topology $S^{*}(I)=\{A \in$ $\left.P(X): C l^{* S}\left(A^{c}\right)=A^{c}\right\}$, which is finer than $\mathrm{S}$.

Proof. (1) By Theorem 3.1.(1), $0_{\sim}^{* S}=0_{\sim}$, we have $C l^{* S}\left(0_{\sim}\right)=$ $0 \sim$

(2) Clear $A \subseteq C l^{* S}$ for every intuitionistic fuzzy set A.

(3) Let $\mathrm{A}$ and, B be two intuitionistic fuzzy sets. Then, $C l^{* S}(A) \cup C l^{* S}(B)=\left(A \cup A^{* S}\right) \cup\left(B \cup B^{* S}\right)=(A \cup B) \cup$ $\left(A^{* S} \cup B^{* S}\right) \subseteq(A \cup B) \cup(A \cup B)^{* S}=C l^{* S}(A \cup B)$ (by Theorem 3.1 (7). Hence, $C l^{* S}(A) \cup C l^{* S}(B) \subseteq C l^{* S}(A \cup B)$.

(4) Let A be any intuitionistic fuzzy set. Since, by (2), $A \subseteq C l^{* S}(A)$, then $C l^{* S}(A) \subseteq C l^{* S}\left(C l^{* S}(A)\right)$. On the other hand, $C l^{* S}\left(C l^{* S}(A)\right)=C l^{* S}\left(A \cup A^{* S}\right)=\left(A \cup A^{* S}\right) \cup(A \cup$ $\left.A^{* S}\right)^{* S} \subseteq A \cup A^{* S} \cup A^{* S}=C l^{* S}(A)$ (by Theorem 3.3), it follows that $C l^{* S}\left(C l^{* S}(A)\right) \subseteq C l^{* S}(A)$. Hence, $C l^{* S}\left(C l^{* S}(A)\right)$
$=C l^{* S}(A)$. Consequently, $C l^{* S}(A)$ is an intuitionistic fuzzy supra closure operator. Also, it is also easy to show that the collection $S^{*}(I)=\left\{A \in P(X): C l^{* S}\left(A^{c}\right)=A^{c}\right\}$ is an intuitionistic fuzzy supra topology on $X$, which is called the intuitionistic fuzzy supra topology induced by the intuitionistic fuzzy supra closure operator.

Example 3.2. For any intuitionistic fuzzy ideal on $\mathrm{X}$ if $I=$ $\{0 \sim\} \Rightarrow C l^{* S}(A)=A \cup A^{* S}=A \cup C l^{S}(A)=C l^{S}(A)$ for every $A \in P(X)$. So $S^{*}\left(\left\{0_{\sim}\right\}\right)=S$, and if $I=P(X) \Rightarrow$ $C l^{* S}(A)=A$, because $A^{* S}=0 \sim$ for every $A \in P(X)$. So $S^{*}(P(X))$ is an intuitionistic fuzzy discrete supra topology on $X$. Since $\{0 \sim\}$ and $P(X)$ are the two extreme intuitionistic fuzzy ideals on $\mathrm{X}$, therefore for any intuitionistic fuzzy ideal I on $\mathrm{X}$, we have $\left\{0_{\sim}\right\} \subseteq I \subseteq P(X)$. So we can conclude by Theorem 3.1.(2) $S^{*}(\{0 \sim\}) \subseteq S^{*}(I) \subseteq S^{*}(P(X))$, i.e. $S \subseteq S^{*}(I)$, for any intuitionistic fuzzy ideal I on X. In particular, we have for any two intuitionistic fuzzy ideals $I_{1}$ and $I_{2}$ on $\mathrm{X}, I_{1} \subseteq I_{2} \Rightarrow$ $S^{*}\left(I_{1}\right) \subseteq S^{*}\left(I_{2}\right)$.

Theorem 3.5. Let $S_{1}, S_{2}$ be two intuitionistic fuzzy supra topologies on X. Then, for any intuitionistic fuzzy ideal I on X, $S_{1} \subseteq S_{2}$ implies

(1) $A^{* S}\left(S_{2}, I\right) \subseteq A^{* S}\left(S_{1}, I\right)$ for every $A \in P(X)$,

(2) $S_{1}^{*}(I) \subseteq S_{2}^{*}(I)$.

Proof. (1) Since every $S_{1}$ s-neighborhood of any intuitionistic fuzzy point $x_{(\alpha, \beta)}$ is also ana $S_{2}$ s-neighborhood of $x_{(\alpha, \beta)}$. Therefore, $A^{* S}\left(S_{2}, I\right) \subseteq A^{* S}\left(S_{1}, I\right)$.

(2) Clearly, $S_{1}^{*}(I) \subseteq S_{2}^{*}(I)$ as $A^{* S}\left(S_{2}, I\right) \subseteq A^{* S}\left(S_{1}, I\right)$.

Theorem 3.6. Let $(X, S, I)$ be an intuitionistic fuzzy ideal supra topological space. Then, A is an intuitionistic fuzzy $S^{*}$ supra closed if and only if $A^{* S} \subseteq A$. Then, $A=C l^{S}\left(A^{* S}\right)=$ $C l^{* S}(A)$.

Proof. Clear.

Theorem 3.7. Let (X, S, I) be an intuitionistic fuzzy ideal supra topological space. Then, the collection $\beta(I, S)=\{U-$ $H: U \in S, H \in I\}$ is a base for the intuitionistic fuzzy supra topology $S^{*}(I)$.

Proof. Let $U \in S^{*}(I)$ and $x_{(\alpha, \beta)} \in U$. Then, $U^{c}$ is an intuitionistic fuzzy $S^{*}$-supra closed set suchso that $C l^{* S}\left(U^{c}\right)=$ $U^{c}$, and hence $\left(U^{c}\right)^{* S} \subseteq U^{c}$. Then, $x_{(\alpha, \beta)} \notin\left(U^{c}\right)^{* s}$, and so there, exists $V \in N\left(x_{(\alpha, \beta)}\right)$ such that $V \cap U^{c} \in I$. putting 
$H=V \cap U^{c}$, then $x_{(\alpha, \beta)} \notin H$, and $H \in I$. Thus, $x_{(\alpha, \beta)} \in V-$ $H=V \cap H^{c}=V \cap\left(V \cap H^{c}\right)^{c}=V \cap\left(V^{c} \cap U\right)=V \cap U \subseteq U$. Hence, $x_{(\alpha, \beta)} \in V-H \subseteq U$, where $V-H \in \beta(I, S)$. Hence, $\mathrm{U}$ is denotes the union of the sets in $\beta(I, S)$.

Theorem 3.8. Let (X, S, I) be an intuitionistic fuzzy ideal supra topological space. Then, $S \subseteq \beta(I, S) \subseteq S^{*}$.

Proof. Let $U \in S$. Then, $U=U-0_{\sim} \in \beta(I, S)$. Hence, $S \subseteq$ $\beta(I, S)$. Now, let $G \in \beta(I, S)$, then there exists $U \in S$ and $H \in I$ such that $G=U-H$. Then, $C l^{* S}\left(G^{c}\right)=C l^{* S}(U-$ $H)^{c}=(U-H)^{c} \cup\left((U-H)^{c}\right)^{* S}=\left(U^{c} \cup H\right) \cup\left(U^{c} \cup H\right)^{* S}$. But $H \in I$, and then by Theorem 3.1.(8), $\left(U^{c} \cup H\right)^{* S}=\left(U^{c}\right)^{* S}$; and so, $C l^{* S}(U-H)^{c}=U^{c} \cup H \cup\left(U^{c}\right)^{* S} \subseteq U^{c} \cup H$. Hence, $C l^{* S}(U-H)^{c} \subseteq U^{c} \cup H=(U-H)^{c}$, but $(U-H)^{c} \subseteq$ $C l^{* S}(U-H)^{c}$. Hence, $C l^{* S}(U-H)^{c}=(U-H)^{c}$. Therefore, $U-H \in S^{*}(I)$. Hence, $\beta(I, S) \subseteq S^{*}(I)$. Consequently, $S \subseteq \beta(I, S) \subseteq S^{*}(I)$.

Theorem 3.9. Let (X, S, I) be an intuitionistic fuzzy ideal supra topological space. Then, if $I=\left\{0_{\sim}\right\}$, then $S=\beta(I, S$ )$=S^{*}(I)$.

Proof. It follows from Theorem 3.8.

Example 3.3. Let $\mathrm{T}$ be the intuitionistic fuzzy indiscrete supra topology on X, i.e. $T=\left\{0_{\sim}, 1_{\sim}\right\}$. So $1_{\sim}$ is the only s-neighborhoods of $x_{(\alpha, \beta)}$. Now, $x_{(\alpha, \beta)} \in A^{* S}$ for an intuitionistic fuzzy set A if and only if for every $U \in N\left(x_{(\alpha, \beta)}\right)$, then $U \cap A \notin I$. So $A \notin I$. Therefore, $A^{* S}=1_{\sim}$ if $A \notin I$ and $A^{* S}=0_{\sim}$ if $A \in I$. This implies that we have $C l^{* S}(A)=$ $A \cup A^{* S}=1 \sim$ if $A \notin I$ and $C l^{* S}(A)=A$ if $A \in I$ for any intuitionistic fuzzy set A of X. Hence, $T^{*}=\left\{M: M^{c} \in I\right\}$. Let $S \cup T^{*}(I)$ be the supremum intuitionistic fuzzy supra topology of $\mathrm{S}$ and $T^{*}(I)$, i.e. the smallest intuitionistic fuzzy supra topology generated by $S \cup T^{*}(I)$. Then, we have the following theorem.

Theorem 3.10. $S^{*}(I)=S \cup T^{*}(I)$.

Proof. Follows from the fact that $\beta$ forms a basis for $S^{*}(I)$.

\section{S-Compatible Intuitionistic Fuzzy Ideal}

Definition 4.1. Let (X, S, I) be an intuitionistic fuzzy ideal supra topological space. We say the $\mathrm{S}$ is $\mathrm{S}$-compatible with the intuitionistic fuzzy ideal I, denoted as $S \sim I$, if the following holds for every intuitionistic fuzzy set A in X, if for every $x_{(\alpha, \beta)} \in A$, there exists $U \in N\left(x_{(\alpha, \beta)}\right)$ such that $U \cap A \in I$, then $A \in I$.

Theorem 4.1. Let (X, S, I) be an intuitionistic fuzzy ideal supra topological space, and the following properties are equivalent;

(1) $S \sim I$,

(2) For every intuitionistic fuzzy set $\mathrm{A}$ in $\mathrm{X}, A \cap A^{* S}=0 \sim$ implies that $A \in I$,

(3) For every intuitionistic fuzzy set $\mathrm{A}$ in $\mathrm{X}, A-A^{* S} \in I$,

(4) For every intuitionistic fuzzy set A in X, if A contains no non-empty intuitionistic fuzzy subset B with $B \subseteq B^{* S}$, then $A \in I$.

Proof. (1) $\Rightarrow(2)$ The proof is obvious.

(2) $\Rightarrow$ (3) For any intuitionistic fuzzy set A in X, $A-A^{* S} \subseteq$ $A$, and $\left(A-A^{* S}\right) \cap\left(A-A^{* S}\right)^{* S} \subseteq\left(A-A^{* S}\right) \cap A^{* S}=0_{\sim}$. By (2), we obtain $A-A^{* S} \in I$.

$(3) \Rightarrow(4)$ By (3), for every intuitionistic fuzzy set A in X, $A-A^{* S} \in I$. Let $A-A^{* S}=E \in I$; then $A=E \cup\left(A \cap A^{* S}\right)$ and by Theorem 3.1.(6) $A^{* S} \subseteq E^{* S} \cup\left(A \cap A^{* S}\right)^{* S}=(A \cap$ $\left.A^{* S}\right)^{* S}$ and $A \cap A^{* S} \subseteq A$ then $\left(A \cap A^{* S}\right)^{* S} \subseteq A^{* S}$ therefore $A^{* S}=\left(A \cap A^{* S}\right)^{* S}$, we have $A \cap A^{* S}=A \cap\left(A \cap A^{* S}\right)^{* S} \subseteq$ $\left(A \cap A^{* S}\right)^{* S}$ and $A \cap A^{* S} \subseteq A$. By the assumption $A \cap A^{* S}=$ $0_{\sim}$, and hence $A=A-A^{* S} \in I$.

$(4) \Rightarrow(1)$ Let an intuitionistic fuzzy set in $\mathrm{X}$ and assume that for every $x_{(\alpha, \beta)}$, there exists $U \in N\left(x_{(\alpha, \beta)}\right)$ such that $U \cap A \in I$. Then, $A \cap A^{* S}=0_{\sim}$. Suppose that A contains B such that $B \subseteq B^{* S}$. Then, $B=B \cap B^{* S} \subseteq A \cap A^{* S}=0_{\sim}$. Therefore, A contains no non-empty subset B with $B \subseteq B^{* S}$. Hence $A \in I$.

Theorem 4.2. Let (X, S, I) be an intuitionistic fuzzy ideal supra topological space. If S is S-compatible with I, then the following equivalent properties hold: ;

(1) For every intuitionistic fuzzy set $\mathrm{A}$ in $\mathrm{X}, A \cap A^{* S}=0_{\sim}$ implies that $A^{* S}=0_{\sim}$,

(2) For every intuitionistic fuzzy set $\mathrm{A}$ in $\mathrm{X},\left(A-A^{* S}\right)^{* S}=$ $0 \sim$.

Proof. First, we show that (1) holds if S is S-compatible with I. Let $\mathrm{A}$ be any intuitionistic fuzzy set in $\mathrm{X}$ and $A \cap A^{* S}=0_{\sim}$. By Theorem 4.1. $A \in I$; then, $A^{* S}=0_{\sim}$.

(1) $\Rightarrow(2)$ Assume that for every intuitionistic fuzzy set A in $\mathrm{X}, A \cap A^{* S}=0_{\sim}$ implies that $A^{* S}=0_{\sim}$. Let $B=A-A^{* S}$, 
then $B \cap B^{* S}=\left(A-A^{* S}\right) \cap\left(A-A^{* S}\right)^{* S}=\left(A \cap\left(A^{* S}\right)^{c}\right) \cap$ $\left.\left(A \cap\left(A^{* S}\right)^{c}\right)^{* S}\right){ }^{s}$ ubseteq $\left((A\right.$ ast Scap $\left.)\left(A^{* S}\right)^{c}\right) \cap\left(A^{* S}\right) \cap$ $\left.\left(\left(A^{* S}\right)^{c}\right)^{* S}\right)=0_{\sim}$. By (1), we have $B^{* S}=0_{\sim}$. Hence, $\left(A-A^{* S}\right)^{* S}=0_{\sim}$.

(2) $\Rightarrow$ (1) Assume that for every intuitionistic fuzzy set A in $\mathrm{X}, A \cap A^{* S}=0_{\sim}$, and let $B=A-A^{* S}$, then $A=B \cup(A \cap$ $\left.A^{* S}\right)=B \cup 0_{\sim}=B$, then $A^{* S}=B^{* S}=\left(A-A^{* S}\right)^{* S}=$ $0_{\sim}$.

Theorem 4.3. Let (X, S, I) be an intuitionistic fuzzy ideal supra topological space, the following properties are equivalent;

(1) $S \cap I=0_{\sim}$,

(2) $1_{\sim}^{* S}=1_{\sim}$.

Proof. (1) $\Rightarrow(2)$ Let $S \cap I=0_{\sim}$. Then $1_{\sim}^{* S}=C l^{S}\left(1_{\sim}\right)=$ $1_{\sim}$.

$(2) \Rightarrow(1) 1_{\sim}=1_{\sim}^{* S}=\left\{x_{(\alpha, \beta)} \in X: U \cap 1_{\sim}=U \notin I\right.$, for every $\left.U \in N\left(x_{(\alpha, \beta)}\right)\right\}$. Hence $C l^{S}(S) \cap I=0_{\sim}$.

\section{Intuitionistic Fuzzy Set Operator $\Psi_{S}$}

Definition 5.1. Let $(X, S, I)$ be an intuitionistic fuzzy ideal supra topological space. An operator $\Psi_{S}: P(X) \rightarrow S$ is defined as follows for every intuitionistic fuzzy set $\mathrm{A}$ in $\mathrm{X}$, $\Psi_{S}(A)=\left\{x_{\alpha, \beta}\right.$ intuitionistic fuzzy point: there exists $M \in$ $N^{S}\left(x_{\alpha, \beta}\right)$ such that $\left.M-A \in I\right\}$. We observe that $\Psi_{S}(A)=$ $1_{\sim}-\left(1_{\sim}-A\right)^{* S}$. The behaviors of the operator $\Psi_{S}$ has been discussed in the following theorem:

Theorem 5.1. Let (X, S, I) be an intuitionistic fuzzy ideal supra topological space. Let A and B be two intuitionistic fuzzy set in X. Then,

(1) $\Psi_{S}(A)$ is intuitionistic fuzzy supra open set.

(2) $\operatorname{Int}^{S}(A) \subseteq \Psi_{S}(A)$.

(3) If $A \subseteq B$, then $\Psi_{S}(A) \subseteq \Psi_{S}(B)$.

(4) $\Psi_{S}(A \cap B) \subseteq \Psi_{S}(A) \cap \Psi_{S}(B)$.

(5) $\Psi_{S}(A) \cup \Psi_{S}(B) \subseteq \Psi_{S}(A \cup B)$.

(6) If $U \in S$, then $U \subseteq \Psi_{S}(U)$.

(7) $\Psi_{S}(A) \subseteq \Psi_{S}\left(\Psi_{S}(A)\right)$.

(8) $\Psi_{S}(A)=\Psi_{S}\left(\Psi_{S}(A)\right)$ if and only if $\left(1_{\sim}-A\right)^{* S}=\left(\left(1_{\sim}\right.\right.$ $\left.-A)^{* S}\right)^{* S}$.

(9) If $(A-B) \cup(B-A) \in I$, then $\Psi_{S}(A)=\Psi_{S}(B)$.
(10) If $E \in I$, then $\Psi_{S}(E)=1_{\sim}-1_{\sim}^{* S}$.

(11) If $E \in I$, then $\Psi_{S}(A-E)=\Psi_{S}(A)$.

(12) If $E \in I$, then $\Psi_{S}(A \cup E)=\Psi_{S}(A)$.

Proof. (1) Since $\left(1_{\sim}-A\right)^{* S}$ is an intuitionistic fuzzy supra closed set, then $1_{\sim}-\left(1_{\sim}-A\right)^{* S}$ is an intuitionistic fuzzy supra open set. Hence, $\Psi_{S}(A)$ is an intuitionistic fuzzy supra open set.

(2) From the definition of the $\Psi_{S}$ operator, $\Psi_{S}(A)=1_{\sim}-$ $\left(1_{\sim}-A\right)^{* S}$. Then, $1_{\sim}-C l^{S}\left(1_{\sim}-A\right) \subseteq 1_{\sim}-\left(1_{\sim}-A\right)^{* S}=$ $\Psi_{S}(A)$, from Theorem 3.1.(4). Hence, $\operatorname{Int}^{S}(A) \subseteq \Psi_{S}(A)$.

(3) Let $A \subseteq B$. Then, $\left(1_{\sim}-B\right) \subseteq\left(1_{\sim}-A\right)$. ThenSubsequently, from Theorem 3.1.(2), $\left(1_{\sim}-B\right)^{* S} \subseteq\left(1_{\sim}-A\right)^{* S}$. Therefore, $\Psi_{S}(A) \subseteq \Psi_{S}(B)$.

(4) We have $A \cap B \subseteq A$ and $A \cap B \subseteq B$. Then from (3), $\Psi_{S}(A \cap B) \subseteq \Psi_{S}(A) \cap \Psi_{S}(B)$.

(5) We have $A \subseteq A \cup B$ and $B \subseteq A \cup B$. Then, from (3), $\Psi_{S}(A) \cup \Psi_{S}(B) \subseteq \Psi_{S}(A \cup B)$.

(6) Let $U \in S$. Then, $\left(1_{\sim}-U\right)$ beis an intuitionistic fuzzy supra closed set, and hence $C l^{S}\left(1_{\sim}-U\right)=\left(1_{\sim}-U\right)$. Then, $\left(1_{\sim}-U\right)^{* S} \subseteq C l^{S}\left(1_{\sim}-U\right)=\left(1_{\sim}-U\right)$. Hence, $U \subseteq$ $1_{\sim}-\left(1_{\sim}-U\right)^{* S}$, and so $U \subseteq \Psi_{S}(U)$.

(7) From (2), $\Psi_{S}(A) \in S$, and from (6), $\Psi_{S}(A) \subseteq \Psi_{S}\left(\Psi_{S}(A)\right)$.

(8) Let $\Psi_{S}(A)=\Psi_{S}\left(\Psi_{S}(A)\right)$. Then $1_{\sim}-\left(1_{\sim}-A\right)^{* S}=$ $\Psi_{S}\left(1_{\sim}-\left(1_{\sim}-A\right)^{* S}\right)=1_{\sim}-\left(1_{\sim}-\left(1_{\sim}-\left(1_{\sim}-A\right)^{* S}\right)^{* S}\right)=$ $1_{\sim}-\left(\left(1_{\sim}-A\right)^{* S}\right)^{* S}$. Therefore, $\left(1_{\sim}-A\right)^{* S}=\left(\left(1_{\sim}-\right.\right.$ $\left.A)^{* S}\right)^{* S}$. Conversely, suppose that $\left(1_{\sim}-A\right)^{* S}=\left(\left(1_{\sim}-\right.\right.$ $\left.A)^{* S}\right)^{* S}$ holds. Then, $1_{\sim}-\left(1_{\sim}-A\right)^{* S}=1_{\sim}-\left(\left(1_{\sim}-A\right)^{* S}\right)^{* S}$ and $1_{\sim}-\left(1_{\sim}-A\right)^{* S}=1_{\sim}-\left(1_{\sim}-\left(1_{\sim}-\left(1_{\sim}-A\right)^{* S}\right)\right)^{* S}=$ $1_{\sim}-\left(1_{\sim}-\Psi_{S}(A)\right)^{* S}$. Hence, $\Psi_{S}(A)=\Psi_{S}\left(\Psi_{S}(A)\right)$.

(9) Let $(A-B) \cup(B-A) \in I$, and let $A-B=E_{1}, B-$ $A=E_{2}$. We observe that $E_{1}, E_{2} \in I$ by heredity, and $B=$ $\left(A-E_{1}\right) \cup E_{2}$. Thus, $\Psi_{S}(A)=\Psi_{S}\left(A-E_{1}\right)=\Psi_{S}((A-$ $\left.\left.E_{1}\right) \cup E_{2}\right)=\Psi_{S}(B)$.

(10) By Theorem 3.1.(9), we obtain if $E \in I$, then $\Psi_{S}(E)=$ $1 \sim-1 \sim$.

(11) This follows from Theorem 3.1.(9), and $\Psi_{S}(A-E)=$ $1_{\sim}-\left(1_{\sim}-(A-E)\right)^{* S}=1_{\sim}-\left(\left(1_{\sim}-A\right) \cup E\right)^{* S}=1_{\sim}-$ $\left(1_{\sim}-A\right)^{* S}=\Psi_{S}(A)$.

(12) This follows from Theorem 3.1.(9), and $\Psi_{S}(A \cup E)=$ $1_{\sim}-\left(1_{\sim}-(A \cup E)\right)^{* S}=1_{\sim}-\left(\left(1_{\sim}-A\right)-E\right)^{* S}=1_{\sim}-$ $\left(1_{\sim}-A\right)^{* S}=\Psi_{S}(A)$.

Theorem 5.2. Let $(\mathrm{X}, \mathrm{S}, \mathrm{I})$ be an intuitionistic fuzzy ideal supra topological space. If $\eta=\left\{A \in P(X): A \subseteq \Psi_{S}(A)\right\}$. Then, $\eta$ is an intuitionistic fuzzy supra topology for $\mathrm{X}$. 
Proof. Let $\eta=\left\{A \in P(X): A \subseteq \Psi_{S}(A)\right\}$. By Theorem 3.1.(1), $0_{\sim}^{* S}=0_{\sim}$ and $\Psi_{S}\left(1_{\sim}\right)=1_{\sim}-\left(1_{\sim}-1_{\sim}\right)^{* S}=$ $1_{\sim}-0_{\sim}^{* S}=1_{\sim}$. Moreover, $\Psi_{S}\left(0_{\sim}\right)=1_{\sim}-\left(1_{\sim}-0_{\sim}\right)^{* S}=$ $1_{\sim}-1_{\sim}=0_{\sim}$. Therefore, we observe obtain that $0_{\sim} \subseteq$ $\Psi_{S}\left(0_{\sim}\right)$ and $1_{\sim} \subseteq \Psi_{S}\left(1_{\sim}\right)=1_{\sim}$, and thus $0_{\sim}, 1_{\sim} \in \eta$. Now, if $\left\{A_{\alpha}: \alpha \in \Delta\right\} \subseteq \eta$, then $A_{\alpha} \subseteq \Psi_{S}\left(A_{\alpha}\right) \subseteq \Psi_{S}\left(\cup A_{\alpha}\right)$ for every $\alpha$; and hence, $\bigcup A_{\alpha} \subseteq \Psi_{S}\left(\bigcup A_{\alpha}\right)$. This shows that $\eta$ is an intuitionistic fuzzy supra topology.

Definition 5.2. An intuitionistic fuzzy ideal I in a space (X, $\mathrm{S}, \mathrm{I})$ is called an S-codense intuitionistic fuzzy ideal if $S \cap$ $I=\left\{0_{\sim}\right\}$. The following theorem is related to the S-codense intuitionistic fuzzy ideal.

Theorem 5.3. Let (X, S, I) be an intuitionistic fuzzy ideal supra topological space and let I be S-codense with S. Then, $1_{\sim}=1_{\sim}^{* S}$.

Proof. It is obvious.

Definition 5.3. Let (X, S, I) be an intuitionistic fuzzy ideal supra topological space. An intuitionistic fuzzy set $\mathrm{A}$ in $\mathrm{X}$ is called a $\Psi_{S}$-C-intuitionistic fuzzy set if $A \subseteq C l^{S}\left(\Psi_{S}(A)\right)$. The collection of all $\Psi_{S}$-C-intuitionistic fuzzy set in (X, S, I) is denoted by $\Psi_{S}(X, S)$.

Theorem 5.4. Let (X, S, I) be an intuitionistic fuzzy ideal supra topological space. If $A \in S$, then $A \in \Psi_{S}(X, S)$.

Proof. From Theorem 5.1.(6) it follows that $S \subseteq \Psi_{S}(X, S)$.

Theorem 5.5. Let $\left\{A_{\alpha}: \alpha \in \Delta\right\}$ be a collection of nonempty $\Psi_{S}$-C-intuitionistic fuzzy sets in the intuitionistic fuzzy ideal supra topological space (X, S, I); then $\bigcup_{\alpha \in \Delta} A_{\alpha} \in \Psi_{S}(X, S)$.

Proof. For each $\alpha \in \triangle$,

$$
A_{\alpha} \subseteq C l^{S}\left(\Psi_{S}\left(A_{\alpha}\right)\right) \subseteq C l^{S}\left(\Psi_{S}\left(\bigcup_{\alpha \in \Delta} A_{\alpha}\right)\right)
$$

This implies that $\bigcup_{\alpha \in \Delta} A_{\alpha} \subseteq C l^{S}\left(\Psi_{S}\left(\bigcup_{\alpha \in \Delta} A_{\alpha}\right)\right)$. Thus $\bigcup_{\alpha \in \Delta} A_{\alpha} \in \Psi_{S}(X, S)$.

\section{Conflict of Interest}

No potential conflict of interest relevant to this article was reported.

\section{References}

[1] L. A. Zadeh, "Fuzzy sets," Information and Control, vol. 8, no. 3, pp. 338-353, 1965. https://doi.org/10.1016/S00199958(65)90241-X

[2] K.T. Atanassov, "Intuitionistic fuzzy sets," Fuzzy Sets and Systems, vol. 20, no. 1, pp. 87-96, 1986. https://doi.org/10. 1016/S0165-0114(86)80034-3

[3] K. T. Atanassov, "New operations defined over the intuitionistic fuzzy sets," Fuzzy Sets and Systems, vol. 61, no. 2, pp. 137-142, 1994.

[4] K. T. Atanasov, Intuitionistic Fuzzy Sets: Theory and Applications. Berlin, Germany: Springr, 1999.

[5] E. Szmidt and J. Kacprzyk, "Intuitionistic fuzzy sets in some medical applications," Notes on IFS, vol. 7, no. 4, pp. 58-64, 2001.

[6] E. Szmidt and J. Kacprzyk, "Medical diagnostic reasoning using a similarity measure for intuitionistic fuzzy sets," Notes on IFS, vol. 10, no. 4, pp. 61-69, 2004.

[7] K. Atanassov, "Review and new results on intuitionistic fuzzy sets," in Mathematical Foundations of Artificial Intelligence Seminar, Sofia, Bulgaria, 1988.

[8] D. Coker and M. Demirci, "On intuitionistic fuzzy points," Notes on IFS, vol. 1, pp. 79-84, 1995.

[9] K. Kuratowski, Topology (Volume I). New York, NY: Academic Press, 1966.

[10] R. Vaidyanathaswamy, "The localisation theory in settopology," Proceedings of the Indian Academy of SciencesSection A, vol. 20, no. 1, pp. 51-61, 1944. https://doi.org/ 10.1007/BF03048958

[11] D. Jankovic and T. R. Hamlett, "New topologies from old via ideals," The American Mathematical Monthly, vol. 97, no. 4, pp. 295-310, 1990. https://doi.org/10.1080/ 00029890.1990 .11995593

[12] A. S. Mashhour, A. A. Allam, F. S. Mahmoud, and F. H. Khedr, "On supra topological spaces," Indian Journal of Pure and Applied Mathematics, vol. 14, no. 4, pp. 502-510, 1983.

[13] N. Turanl, "On intuitionistic fuzzy supra topological spaces," in Proceedings of International Conference on Modeling and Simulation, Spain, 1999, pp. 69-77. 
[14] A. Kandil, O. A. Tantawy, S. A. El-Sheikh, and S. A. Hazza, "New supra topologies from old via ideals," Journal of New Theory, vol. 2015, no. 4, pp. 1-5, 2015.

[15] K. T. Atanassov, "Intuitionistic fuzzy sets," in VII ITKR's Session, Sofia (deposed in Central Sci.-Technical Library of Bulg. Acad. of Sci., 1697/84). Sofia, Bulgaria: Bulgarian Academy of Sciences, 1984.

[16] B. Riecan and K. T. Atanassov, "Some properties of two operations over intuitionistic fuzzy sets," Notes Intuit Fuzzy Sets, vol. 14, pp. 6-10, 2008.

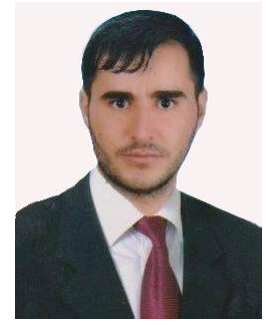

Fadhil Abbas is an Ph.D. student at Johannes Kepler University, Linz, Austria since March 2018. He worked as an Assistant at the University of Telafer. He completed his Master's degree from Gazi University in Fuzzy Topology in 2011. His current research interests include fuzzy topology and intuitionistic fuzzy topology, etc. He has has published more than three articles.

E-mail: fadhilhaman@gmail.com 